A theoretical interpretation of the main scrape-off layer heat-flux width scaling for tokamak inner-wall limited plasmas

This content has been downloaded from IOPscience. Please scroll down to see the full text.

View the table of contents for this issue, or go to the journal homepage for more

Download details:

This content was downloaded by: riccipaolo

IP Address: 128.179.254.59

This content was downloaded on 09/07/2016 at 06:45

Please note that terms and conditions apply. 


\title{
A theoretical interpretation of the main scrape-off layer heat-flux width scaling for tokamak inner-wall limited plasmas
}

\author{
F D Halpern ${ }^{1}$, J Horacek ${ }^{2}$, R A Pitts ${ }^{3}$ and P Ricci ${ }^{1}$ \\ ${ }^{1}$ École Polytechnique Fédérale de Lausanne (EPFL), Centre de Recherches en Physique des Plasmas \\ (CRPP), CH-1015 Lausanne, Switzerland \\ ${ }^{2}$ Institute of Plasma Physics ASCR, Prague, Czech Republic \\ ${ }^{3}$ ITER Organization, Route de Vinon-sur-Verdon CS 90 046, F-13067 St Paul lez Durance Cedex, France \\ E-mail: federico.halpern@epfl.ch
}

Received 30 September 2015, revised 8 May 2016

Accepted for publication 26 May 2016

Published 5 July 2016

\begin{abstract}
The International Tokamak Physics Activity Topical Group on scrape-off layer and divertor physics has amassed a database comprising hundreds of reciprocating Langmuir probe measurements of the main scrape-off layer heat-flux width $\lambda_{q}$ in inner-wall limited discharges. We have carried out an analysis, based on turbulent transport theory, of the variation of $\lambda_{q}$ with respect to the dimensionless plasma parameters. Restricting our analysis to circular plasmas, we find that a model based on non-linearly saturated turbulence can well reproduce the $\lambda_{q}$ values found in the database.
\end{abstract}

Keywords: edge plasma, heat-flux width, scrape-off layer

(Some figures may appear in colour only in the online journal)

\section{Introduction}

Since the ramp-up phase of ITER plasmas are expected to be mostly inner-wall limited (IWL) [1], it is important to establish a predictive capability describing the scrape-off layer (SOL) heat-flux width, $\lambda_{q}=-q_{\|} / \nabla q_{\|}\left(q_{\|} \sim n c_{s} T\right)$, in this configuration. The original ITER heat-load specifications assumed that $\lambda_{q}$ in IWL discharges would follow a powerlaw scaling originally obtained for L-mode diverted plasmas [2]. In recent years, however, this assumption has been clearly shown to be flawed.

An extensive study of limiter discharges in Tore Supra and JET demonstrated that the scaling was not obeyed in these devices [3]. Stimulated by these observations, the scrape-off-layer and divertor topical group of the International Tokamak Physics Activity (ITPA) embarked on a multi-machine database effort to characterize the main SOL $\lambda_{q}$ and confirm the choices made by the ITER Organization (IO) in the design of the ITER inner-wall toroidal shaping [1, 4-6]. As part of this effort, dedicated experiments on JET subsequently found clear evidence of a narrow feature in the SOL heat flux close to the last closed flux surface (LCFS), although there was insufficient data to obtain a scaling for either the near-SOL feature or the broader profile width in the main SOL [7]. These JET experiments, in turn, provoked a new multi-machine effort to investigate this narrow feature [8-11], ultimately leading to a re-design of the ITER inner-wall toroidal profile shape taking the narrow feature into account [12]. The main SOL heat-flux database obtained, as well as the scalings derived from the ITPA study, are described in a companion paper of this special issue [13].

The database is comprised of hundreds of reciprocating Langmuir probe measurements performed in IWL discharges in a large number of tokamaks. Each reciprocation is fitted using an exponential power law with a single decay length, i.e. neglecting the narrow feature, which is rather challenging to measure using fast probes and which is best inferred from infrared thermography of the limiter surface [12].

Within the companion paper, only known ITER parameters, such as SOL power, toroidal field $B_{\phi}$, major radius 
$R_{0}$, plasma current $I_{\mathrm{p}}$, safety factor $q$, etc, were considered as regression parameters in order to minimize the error in the possible scalings. The principal finding of the study is that several scalings can be constructed from these engineering parameters, all with similar coefficients of determination ( $R^{2}$ parameter). Fortunately, all scalings project approximately the same main SOL $\lambda_{q}$ for ITER inner-wall start-up plasmas. However, a straightforward physical interpretation of the data is not possible.

The aim of the present work is, in fact, to assess the main SOL $\lambda_{q}$ from a theoretical perspective. In an attempt to seek a physics-based understanding of this database, we have reassessed its contents from a completely different perspective. Rather than concentrating on finding suitable scaling parameters directly from known ITER quantities, our approach is to use SOL turbulent transport theory to guide our choice of parameters. In this approach, the scaling parameters are dimensionless. A priori, it may seem like this approach has a serious disadvantage. The dimensionless plasma parameters required by the theory involve the last closed flux surface (LCFS) local temperature and density, and are more difficult to determine from experimental reciprocating Langmuir probe measurements. The latter are often noisy and subject to systematic error, particularly as the probes penetrate deeper into the SOL where the profile steepens rapidly. In addition, the location of the LCFS is based on magnetic equilibrium reconstruction, which is precise to a few millimeters, and the LCFS is often not reached by the probes. As we will show, however, the experimental uncertainty related to these parameters has little effect on the final result.

Despite the uncertainties introduced by requiring absolute values of local plasma parameters, our approach has advantages and some beneficial side effects. First, the theoretical analysis is not constrained by mutual correlations between the regression parameters, which limits the combination of parameters available in [13]. Second, the ITPA database allows recently proposed theoretical models to be tested $[14,15]$. Third, it is possible to apply hybrid theory/data analysis to the database, leading to new, possibly more precise, descriptions of the SOL width. Fourth, and most important, our approach allows us (for a subset of the data, at least) to better interpret and understand the physical origin of the variation of $\lambda_{q}$ with the plasma parameters.

At the time the database was being compiled, there was no theory-based SOL model with credible predictive capability for the main SOL $\lambda_{q}$. Since then, 3D non-linear, fluxdriven turbulence simulations of SOL dynamics have been revealed that, (a) the turbulent modes saturate through the gradient removal mechanism [14], (b) turbulence is driven by ballooning or drift type modes [16, 17], and (c) the transport levels are strongly affected by parallel dynamics effects, such as collisionality and electromagnetic flutter, and by the normalized plasma size $[18,19]$. The combination of these elements led to resistive ballooning mode (RBM) scaling [15], which compared favorably against a small experimental database gathered from existing published SOL widths in limiter discharges. The work presented here shows, in fact, that the mechanisms set forward in [14-17, 19], describing main SOL transport dynamics in circular IWL discharges, are consistent with the ITPA database.

\section{On the choice of model and parameters}

The cold-ion electrostatic drift-reduced Braginskii equations [20, 21], expressed for circular flux-surface geometry (see appendix), are used as the basis of our analysis. Since it is typically observed that $T_{i}>T_{e}$ in the SOL [22], we comment on the neglect of $T_{i}$ in the model. The effects of finite $T_{i}$ are exhaustively analyzed for the IWL configuration by Mosetto et al [17], finding altogether a weak contribution to the SOL transport dynamics. Purely ion-temperature-driven modes, such as the ion temperature gradient (ITG) instability, are ruled out. The only noteworthy effect, at transport-relevant wavenumbers, is a slight enhancement of the RBM instability leading to a factor of $\left(1+T_{i} / T_{e}\right)^{1 / 7}$ in the final expression of the RBM scaling [15]. More recently, Manz et al [23] have pointed out the importance of $T_{i}$ effects for blob motion in certain regimes, which could also affect the gradient lengths.

The weak influence found for $T_{i}$ effects in turbulence simulations, combined with the lack of $T_{i}$ profiles in the database, motivate our choice of a cold ion model. The effects of $T_{i}$ could be easily reintroduced in the present work, if $T_{i}$ data became available. The main qualitative parametric trends and conclusions of the study should remain unaffected, although details of the regression fits may be altered.

To obtain the final form of the drift-reduced Braginskii equations shown in the appendix, we follow [21], where temperature and density are normalized to their values at the LFCS, $T_{e, \mathrm{LCFS}}$ and $n_{\mathrm{LFCS}}$, and we choose a reference perpendicular length $L_{\perp}=\rho_{s}=c_{s} / \omega_{c i}\left(c_{s}=\sqrt{T_{e, \mathrm{LCFS}} / m_{i}}, \omega_{c i}=e B_{\phi} / m_{i}\right)$, a reference parallel length $L_{\|}=R_{0}$ (the major radius), and a reference time $\tau_{\text {ref }}=R_{0} / c_{s}$ (here we use SI units to define these physical quantities, except the temperature which is expressed in eV). Normalization and linearization of the drift-Braginskii equations naturally yields the following set of dimensionless parameters:

$$
\begin{gathered}
\rho_{\star}=\rho_{s} / R_{0} \\
\nu=\frac{e^{2} n_{\mathrm{LCFS}} R_{0}}{m_{i} c_{s} \sigma_{\|}} \\
q \approx q_{95} \sim \frac{a}{R_{0}} \frac{B_{\phi}}{B_{\theta}}
\end{gathered}
$$

where $\sigma_{\|}$is the parallel Spitzer conductivity given in $(\mathrm{Ohm}-\mathrm{m})^{-1}$ and assuming a pure deuterium plasma.

Here, we have replaced $q$ at the LCFS, which appears in the theory, with $q_{95}$, which is the quantity available in the database $^{4}$. The parameters describe, in dimensionless form, the plasma size $\left(\rho_{\star}\right)$, the Spitzer resistivity $(\nu)$, and the connection length $\left(q_{95}\right)$. The Spitzer resistivity is considered

\footnotetext{
${ }^{4}$ Replacing $q_{95}$ with $q$ at the LCFS calculated using a cylindrical formula including elongation introduces no discernible difference in the results of the analysis.
} 
Table 1. Plasma parameters for the devices used in the study.

\begin{tabular}{llllllll}
\hline Device & $R_{0}(\mathrm{~m})$ & $a(\mathrm{~m})$ & $I_{\mathrm{p}}(\mathrm{kA})$ & $B_{\phi}(\mathrm{T})$ & $q_{95}$ & $\kappa$ & No. entries \\
\hline Tore Supra & 2.20 & 0.65 & $500-1200$ & $2.6-4.1$ & $2.9-9.9$ & 1 & 120 \\
DIII-D & 1.70 & 0.60 & $600-1200$ & 1.9 & $2.6-5.1$ & $1.4,1.5$ & 23 \\
COMPASS & 0.55 & 0.20 & $80-180$ & 1.15 & $2.5-13.1$ & 1 & 84 \\
JET & 2.80 & 0.98 & 1500,2500 & 2.8 & $2.5-5.1$ & 1.3 & 27 \\
CASTOR & 0.40 & 0.08 & 9 & 1.3 & 10.8 & 1 & 3 \\
EAST & 1.85 & 0.46 & 300 & 1.96 & 4.2 & 1.15 & 2 \\
HL-2A & 1.67 & 0.36 & $100-220$ & 1.36 & $2.5-5.9$ & 1 & 39 \\
KSTAR & 1.78 & 0.47 & 400 & 2.0 & 4.6 & 1.49 & 1 \\
C-Mod & 0.68 & 0.22 & $400-1100$ & $4-7$ & $2.5-4.25$ & $1.1-1.3$ & 18 \\
\hline
\end{tabular}

Note: Adapted from Horacek et al [13].

since it affects the linear stability of drift and ballooning modes. The normalized collisionality $\nu_{\star}$ can be neglected since perpendicular transport is typically anomalous in the far SOL, with trapped particle effects, in particular, being negligible.

If the main SOL heat-flux widths are solely determined by turbulent transport, these parameters should fully explain the variation of $\lambda_{q}$ in the database. There is a total of 317 entries for which these dimensionless quantities can be computed, including 120 entries from Tore Supra, 23 from DIII-D, 84 from COMPASS, 27 from JET, 3 from CASTOR, 2 from EAST, 39 from HL-2A, 1 from KSTAR, and 18 from Alcator C-Mod. All of these devices used the inner-wall tiles as a limiter (e.g. a toroidally continuous limiter), with the exception of CASTOR, which has a single continuous poloidal ring limiter, and JET, which has a series of poloidal inner-wall guard limiters. The impact of the continuity of the limiter structure on the heat-flux channel has been studied by Stangeby [24]. The parameter ranges for each device are shown in table 1. Entries from other tokamaks are neglected due to the local $n_{\mathrm{LCFS}}$ and $T_{e, \mathrm{LFCS}}$ data not being compiled in the database. We include both circular $(\kappa<1.2)$ and shaped discharges in the analysis, which allows us to indirectly evaluate the importance of shaping in the transport dynamics.

We have also introduced $L_{q}=\lambda_{q} / \rho_{s}$, a dimensionless heatflux width, under the assumption that

$$
\lambda_{q} \propto L_{p}=-p / \nabla p
$$

This assumption is necessary because the database does not contain $L_{p}$, which is the quantity predicted by the theory. For the CASTOR and COMPASS tokamaks we show $\lambda_{I_{\text {sat }}}=-I_{\text {sat }} / \nabla I_{\mathrm{sat}}\left(I_{\mathrm{sat}} \sim n c_{S}\right.$ is the ion saturation current $)$, $\lambda_{T}=-T / \nabla T$, and $\lambda_{p}=-p / \nabla p$ in figure 1 . We find that $\lambda_{p}$ and $\lambda_{q}$ are roughly proportional, with $\lambda_{p} \approx 1.2 \lambda_{q}$. Supported by this finding, we introduce a proportionality constant between $L_{q}$ and $L_{p}$ through a best fit between the theoretical calculations and the experimental data, which always remains within order unity.

Some comments should be made regarding the use of a purely turbulent transport model. A possible caveat in the transport model is the neglect of neutral particles, which could drive parallel temperature gradients. However, we

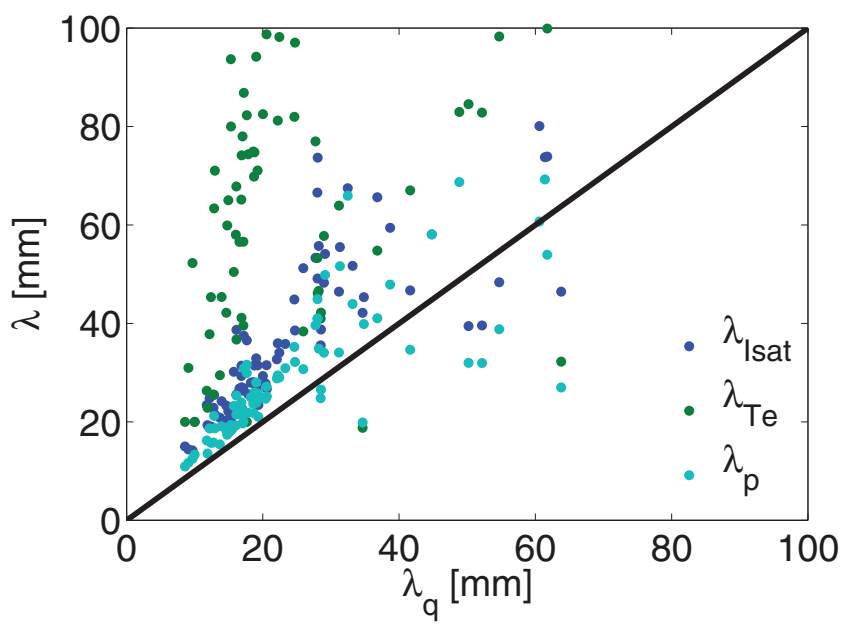

Figure 1. The profile lengths $\lambda_{I_{\text {sat }}}, \lambda_{T}$, and $\lambda_{p}$ are shown for the CASTOR and COMPASS tokamaks. The pressure profile widths, $\lambda_{p}$, are about $20 \%$ longer than the heat-flux widths $\lambda_{q}$.

have approximated the neutral collision length to be at least $8 \lambda_{q}$ at the LCFS of the discharges in the database. Therefore, neutral particles should ionize well inside the plasma and fuel the SOL as they are expelled through the LCFS.

Another possible concern is the lack of the effects of impurities in the theory, in particular, to describe $\lambda_{q}$ in machines with carbon walls. This effect could also lead to parallel temperature gradients, which would translate to a poloidal dependence of $\lambda_{q}$ in the measurements. In recent experiments carried out in Alcator C-Mod, which is a highdensity, high- $Z$ wall device, $\lambda_{q}$ was found to be poloidally uniform $[25]^{5}$. In fact, the poloidal angle at which the Langmuir probe measurements are carried out does not appear to be an important factor in the scalings reported by Horacek et al [13]. We proceed thus, under the assumption that parallel temperature gradients are small. In the end this choice is vindicated by the good agreement between theory and experiment found.

\footnotetext{
${ }^{5}$ The lack of carbon in C-Mod means potentially lower impurity radiation in the contact area, since molybdenum sputters at a higher temperature than carbon. On the other hand, high density should increase the importance of heat conduction compared to convection.
} 

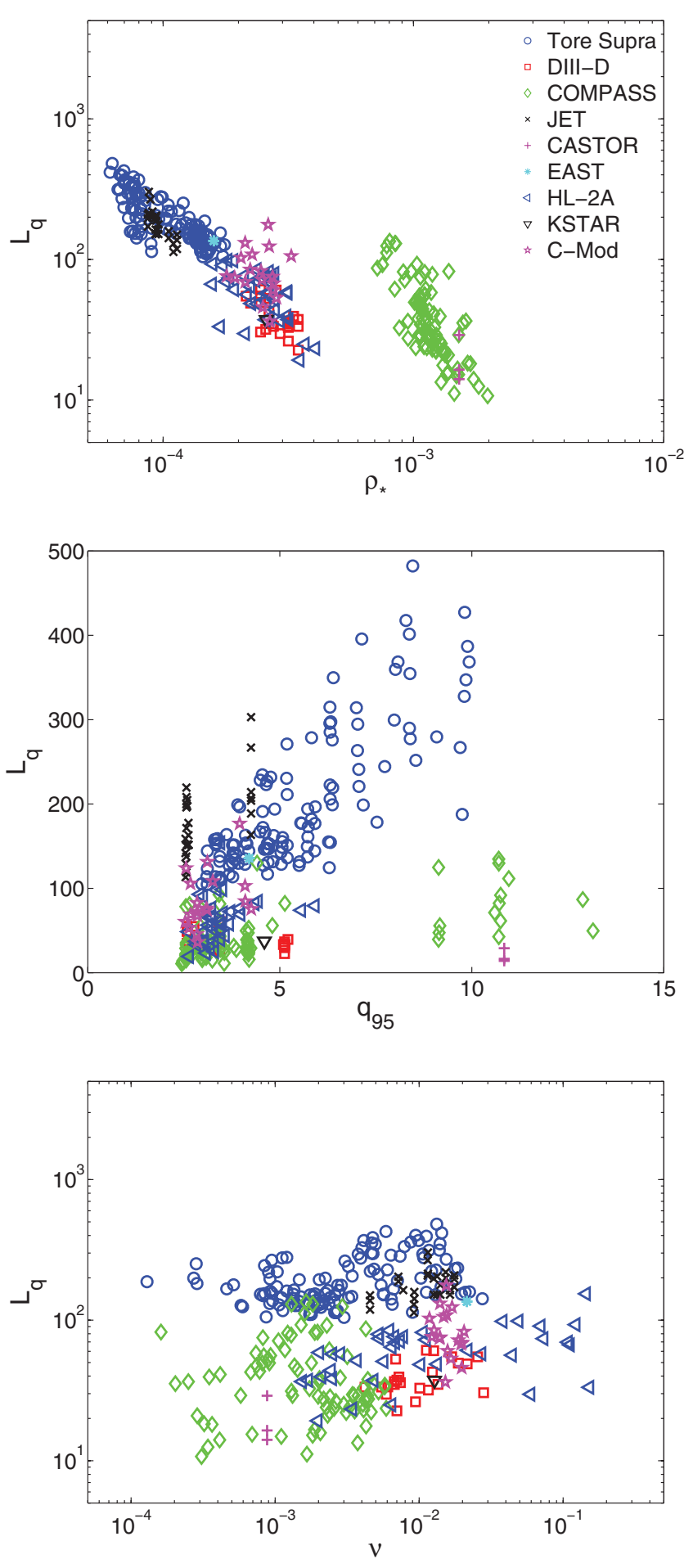

Figure 2. Variation of $L_{q}$ with respect to $\rho_{\star}$ (top), $q_{95}$ (center), and $\nu$ (bottom).

\section{Variation of $L_{q}$ with the dimensionless parameters}

Here, we concentrate on correlating the main SOL dimensionless width $L_{q}=\lambda_{q} / \rho_{s}$, which is the relevant profile scale length in the turbulent transport theory, with respect to the dimensionless plasma parameters $\rho_{\star}, q_{95}$ and $\nu$, described above. This is to demonstrate that correlations between $L_{q}$ and the dimensionless plasma parameters are possible, and to motivate the theory analysis in the following section. A thorough statistical analysis taking into account engineering and geometry parameters (such as $R_{0}, a, I_{\mathrm{p}}, \kappa, a / R_{0}$, etc) has been carried out in a separate paper appearing in this issue [13]. The poloidal gyroradius has been suggested as a scaling parameter for the narrow heat-flux feature $\lambda_{q}$ (which is not addressed by this work), as described by Goldston [26].

At first sight, it appears that $L_{q}$ is correlated with two of the dimensionless parameters. Figure 2 illustrates the variation of the $L_{q}$ with respect to $\rho_{\star}, q_{95}$, and $\nu$. In each panel, we show a scatter plot of $L_{q}$ as a function of one dimensionless parameter. The top panel suggests a powerlaw dependence of $L_{q}$ with respect to the dimensionless plasma size. The datasets with the largest domain in $q_{95}$, Tore Supra and COMPASS, suggest a possible correlation of $L_{q}$ on $q_{95}$. We suspect a predominantly linear dependence with different proportionality constants for each device, which can originate from neglecting other fitting parameters within the scatter plot. Provided that the dependence was linear, it would resemble the $\sim I_{\mathrm{p}}^{-1}$ scaling found both in the RBM scaling for IWL discharges and also for the scaling for H-mode plasma [27]. Additionally, for the COMPASS data, a very large range of $L_{q}$ is possible for essentially the same $\rho_{\star}$. The large scatter seen in the bottom panel supports a weak or zero dependence of $L_{q}$ on $\nu$, as anticipated by the theory $[15,19]$. However, many entries in the database have $\nu \sim 10^{-3}$ and below, which is an order of magnitude smaller than the value assumed in [15]. This implies that inertial effects could become important in the transport theory. The values of $\beta_{e}=2 \mu_{0} p_{e 0} / B_{\phi}^{2}$ and $\alpha=q_{95}^{2} \beta_{e} /\left(\rho_{\star} L_{q}\right)$ are small for all entries in the database, and therefore it is appropriate to neglect electromagnetic effects.

\section{Transport theory and quasi-linear modeling}

In order to compute $L_{q}$, we consider a simple transport equation for $p$ involving only the leading order terms in the pressure balance equation in steady state, $\nabla_{\perp} \cdot\left(p v_{\mathbf{E} \times \mathbf{B}}\right) \sim \nabla_{\|} \cdot\left(p c_{s}\right)$. The dominant fluxes involve $\mathbf{E} \times \mathbf{B}$ cross-field transport driven by non-linearly saturated mesoscale turbulence, $\sim \gamma p /\left(k_{\theta} L_{p}\right)$, where $\gamma$ is the linear growth rate of the turbulent mode and $k_{\theta}$ is the poloidal wavenumber, and sheath losses $\sim p c_{s} / q_{95}$. In deducing the relationship between pressure and potential fluctuations, we assume that the effects of sheared flows are negligible. This might not be valid in the narrow feature region, where shear flow might play an important role [28]. Under these assumptions, the balance of these fluxes gives the gradient removal SOL width

$$
L_{q, \mathrm{gr}} \propto L_{p, \mathrm{gr}}=\frac{q_{95}}{c_{s}}\left(\frac{\gamma}{k_{\theta}}\right)_{\max },
$$

which is valid under the assumption of small parallel temperature gradients. A discussion of the possible turbulent transport mechanisms, as well as the resulting $\lambda_{q}$ stemming from each choice, can be found in [29], while several scalings using 



Figure 3. Resistive ballooning mode theory (top, equation (7)), quasi-linear transport theory (center, equation (8)), and direct fit of $L_{q}$ using dimensionless parameters (bottom, equation (9)) are compared against the heat-flux widths expressed as $L_{q}=\lambda_{q} / \rho_{s}$ (left) and $\lambda_{q}$ (m) (right).

neoclassical transport coefficients for parallel conduction were obtained by Militello et al using the ESEL code [30].

First, we consider the particular case of non-linearly saturated RBM turbulence. RBMs are chosen as a hypothesis for IWL SOL transport because quasi-linear and nonlinear calculations demonstrated clear evidence of RBMs dominating transport at IWL-relevant parameters $(q=3-8$, $\nu=0.01$, weak magnetic shear) [16]. A solution for equation (5) can be obtained analytically for RBMs as follows $[15,19]$. First, a dispersion relation for RBMs is obtained from the reduced resistive MHD equations. Then, the maximum flux that can be driven by RBMs is found by maximizing $\gamma / k_{\theta}$ that appears in equation (5), i.e. we solve $\partial_{k_{\theta}}\left(\gamma / k_{\theta}\right)=0$ starting from the dispersion relation. After straightforward algebra, we find that the flux is maximized for $\gamma=\gamma_{\mathrm{RBM}} \approx \sqrt{2 /\left(\rho_{\star} L_{p}\right)}$ and $k_{\theta}=k_{\theta, \mathrm{RBM}}=\nu^{-1 / 2} q^{-1} \gamma_{\mathrm{RBM}}^{-1 / 2}$. Using $\gamma_{\mathrm{RBM}}$ and $k_{\theta, \mathrm{RBM}}$ in equation (5) leads to the following expression:

$$
L_{p, \mathrm{RBM}}=2^{3 / 7} \nu^{2 / 7} \rho_{\star}^{-3 / 7} q_{95}^{8 / 7} .
$$

We then carry out a least-squares fit to the database to find a proportionality constant between $L_{p}$ and $L_{q}$. The heat-flux widths are given by the expression

$$
L_{q, \mathrm{RBM}} \approx 1.73 L_{p, \mathrm{RBM}}
$$

The top panels of figure 3 show a comparison between equation (7) and the experimental database. On the left 

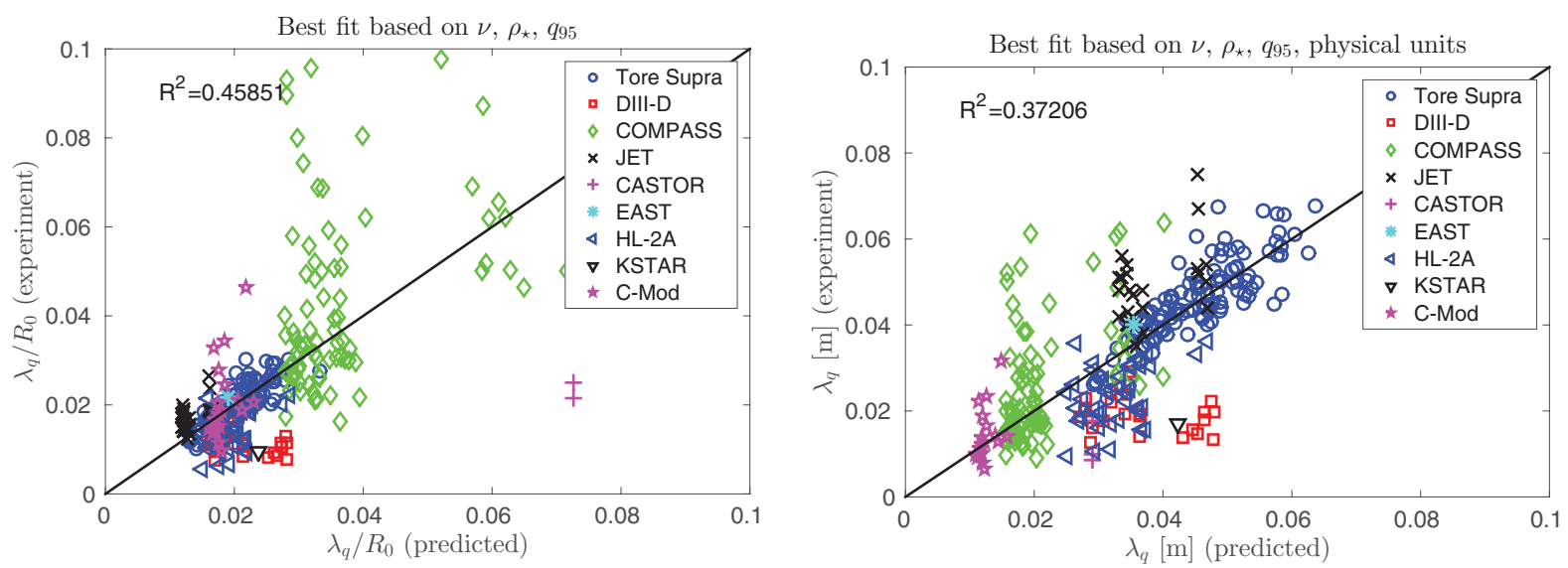

Figure 4. Non-linear regression of $\lambda_{q} / R_{0}$ as a function of $\nu, \rho_{\star}$, and $q_{95}$ is compared against the experimental data, using $R_{0}$ as a unit length (left panel) and using physical units (right panel). The fit results roughly translate into equation (9).

figure, we compare the normalized heat-flux widths $L_{q}$ with the theoretical predictions, while on the right figure the same comparison is repeated, showing $\lambda_{q}=L_{q} \rho_{s}(\mathrm{~m})$. The quality of the comparison is given by $R^{2}$, which is defined here as the square of the Pearson correlation coefficient. The normalized flux-widths from equation (7) match the database quite well with $R^{2}=0.73$, which in actually increases to $R^{2}=0.81$ for nearly circular discharges $(\kappa<1.2$. Shaping effects are not considered in [15]). This level of agreement is as good as the agreement found between equation (6) and non-linear turbulence simulations in our previous work. One possible issue, however, is that the range of $L_{q}$ is dominated by Tore Supra data, which have the greatest variation of main SOL widths, and whose configuration is best captured by our model.

The absolute values of $\lambda_{q}$, on the other hand, are not well described by the resistive ballooning mode theory. Previous non-linear simulation results already hinted at this result: when $\nu<10^{-2}$, inertial effects can become important and therefore the RBM hypothesis must be relaxed.

We have obtained a more precise model where we consider transport driven by all possible unstable modes. Since an analytical solution is not possible, we developed a quasilinear transport code to solve equation (5). The procedure is presented and verified against non-linear simulations in [19]. Only the dimensionless parameters (e.g. $\rho_{\star}, q_{95}$, and $\nu$ ), are needed as inputs. The solution involves computing $\gamma / k_{\theta}$ using the linear version of global Braginskii solver [31] to find the maximum flux. Then, $\left(\gamma / k_{\theta}\right)_{\max }$ is compared to $c_{s} L_{p} / q_{95}$, and $L_{p}$ is adjusted iteratively using a secant method. The iteration stops when left- and right-hand sides of equation (5) match to the desired precision.

Physically, the resulting quasi-linear model obtains $L_{q}$ under the assumptions that perpendicular transport results from nonlinearly saturated turbulence, while parallel transport results from sheath losses. Since the transport calculation is coupled to a complete linear implementation of the drift-Braginskii equations, the turbulent modes can be of drift or ballooning type, with either inertia or resistivity breaking adiabaticity. Different turbulent regimes involving these instabilities, under the same assumptions used in this work, were analyzed in detail by
Mosetto et al $[16,17]$. The most relevant turbulent regimes for the IWL configuration were found to be those where RBMs and inertial drift waves dominated transport.

In what follows, the database entries are used as samples to calculate $L_{p}$ in $\left\{\rho_{\star}, q_{95}, \nu\right\}$ parameter space with the quasilinear code. Then, we employ a robust regression procedure $[32]^{6}$ to obtain a power-law scaling describing the variation of the quasi-linear $L_{p}$ results with the plasma parameters. As before, $L_{p}$ is adjusted by a single constant to obtain $L_{q}$, which is given by

$$
L_{q, \mathrm{QL}}=0.22 \nu^{0.07 \pm 0.01} \rho_{\star}^{-0.62 \pm 0.03} q_{95}^{0.84 \pm 0.03} .
$$

The error in the indicial powers indicates a $95 \%$ confidence interval stemming from the fitting procedure [32]. The comparisons between equation (8) and the experimental data are shown in the center panels of figure 3 . The center-left panel shows good agreement between the quasi-linear computations and the database, particularly for the circular discharges. Once again, the range is dominated by Tore Supra entries, which are very well matched by our computation. As before, the scatter increases when comparing $\lambda_{q}$ rather than $L_{q}$, but for circular discharges we obtain $R^{2}=0.60$, which is comparable to the fit qualities obtained in [13]. For shaped discharges, on the other hand, we find poor agreement between the quasi-linear theory and the experiment.

A careful analysis of the quasi-linear scaling was carried out in order to identify the minimal model equations that yield equation (8). The quasi-linear computation was repeated several times, carefully choosing terms that are known to influence the dynamics of resistive and inertial drift and ballooning modes. It was found necessary to retain most terms of the drift-Braginskii equations, with resistive/inertial drift and ballooning modes all being important in determining the SOL width. This is a result of the large range in

${ }^{6}$ Robust non-linear regression algorithms are more reliable than leastsquares regression when treating noisy sets of data. This is achieved in part by iteratively adjusting the weights of outlier points to increase the fit quality. 
the parameters $\rho_{\star}, \nu$, and $q_{95}$, all of which play an important role in determining the dominant linear instability.

Finally, a scaling for $L_{q}$, as a function of $\nu, \rho_{\star}$, and $q_{95}$, is obtained directly from the data using robust non-linear regression of the circular discharges in the database, which gives

$$
L_{q, \mathrm{fit}}=0.094 \nu^{0.02 \pm 0.02} \rho_{\star}^{-0.71 \pm 0.05} q_{95}^{0.76 \pm 0.06} .
$$

The comparison against experimental data is shown in the bottom panels of figure 3 . The center and bottom panels of figure 3 are quite similar, which is to be expected since expressions (8) and (9) have essentially the same exponents.

Note that the use of $\rho_{s}$ as a reference length may introduce a normalization bias in the Pearson correlation coefficients on the left panels of figure 3 . We have verified that a nonlinear regression of $\lambda_{q} / R_{0}$ as a function of the same dimensionless parameters (figure 4 ) leads to a model equivalent to equation (9). In effect, our normalization length $\rho_{s}$ helps to emphasize database entries with large $\rho_{\star}^{-1}$. Since the dimensionless machine-size scaling of the SOL width remains a significant issue, this effect is in fact desirable.

\section{Conclusion}

In conclusion, straightforward analysis of the ITPA database for the IWL main SOL heat-flux widths reveals that a quasi-linear transport theory (equation (8)) can produce the same regression fit quality as a brute force non-linear fitting procedure based on the same parameters. It can even achieve a similar degree of accuracy as that obtained by engineering parameter scalings using many more regression parameters. We find, however, that the fitting range is dominated by Tore Supra data. This is also the device whose configuration is best described by our model.

The agreement between theory and measurements is very good for circular discharges, which was the scenario considered by the theory, but poor for shaped discharges. For this reason, we have not applied the obtained scaling to ITER discharges, which have elongated and triangular plasma cross-sections. On the other hand, we confirm the $\lambda_{q} \sim I_{\mathrm{p}}^{-1}$ dependence found by many authors, which in our model stems from a combination of parallel dynamics effects and a shorter connection length. Rough estimates ignoring shaping project $\lambda_{q}$ to be within the expected and tolerable range of a few $\mathrm{cm}$.

Previous theoretical studies for edge turbulence in closed field line configurations (e.g. [33]) have shown that turbulent transport decreases when elongation or triangularity increases. Extrapolating these results to SOL turbulence, it appears that shaping should induce order unity modifications to $\lambda_{q}$. In fact, we expect that $\lambda_{q}$ should decrease with increasing elongation or triangularity. However, the precise combination of effects affecting transport, namely, linear stability, field line length, and flux surface area, is rather intricate. Consequently, the precise trends need to be forcibly extracted and interpreted with the aid of nonlinear SOL turbulence simulations, and will be the subject of future work.

The main result of this work can be summarized by expressing equations (8) and (9) in physical units $\left(\mathrm{m}^{-3}, \mathrm{eV}, \mathrm{m}, \mathrm{T}\right)$ :

$$
\begin{aligned}
\lambda_{q, \mathrm{QL}}= & 4.96 \times 10^{-4} n_{\mathrm{LCFS}}^{0.07 \pm 0.01} T_{e, \mathrm{LCFS}}^{0.06 \pm 0.06} R_{0}^{0.68 \pm 0.03} q^{0.84 \pm 0.03} \\
& \times B_{\phi}^{-0.38 \pm 0.02}(\mathrm{~m})
\end{aligned}
$$

$$
\begin{aligned}
\lambda_{q, \mathrm{fit}}= & 2.83 \times 10^{-3} n_{\mathrm{LCFS}}^{0.02 \pm 0.02} T_{e, \mathrm{LFCS}}^{0.10 \pm 0.06} R_{0}^{0.73 \pm 0.07} q^{0.76 \pm 0.06} \\
& \times B_{\phi}^{-0.29 \pm 0.05}(\mathrm{~m}) .
\end{aligned}
$$

The variation shown here for the indicial exponents indicates the $95 \%$ confidence interval of the non-linear fit, but excludes the probe measurement error in $n_{e, \mathrm{LCFS}}$ and $T_{e, \text { LCFS }}$. Readers are also directed to the work of Connor [34], Militello [30], and Myra [29], where $\lambda_{q}$ is studied using analytical theory and 2D non-linear flux-driven simulations. The strong $q_{95}$ and $R_{0}$ dependencies, also recovered by Militello and by Myra, appear to be generic features of SOL transport resulting from the ballooning drive $\left(R_{0}\right)$ and from the parallel mode structure and parallel convection $\left(q_{95}\right)$. We have numerically confirmed that equation (11) represents an improvement with respect to formulas (21) and (22) in [30]. We believe that this improvement arises mainly due to the inclusion of 3D effects in our work, e.g. taking into account the parallel structure of ballooning modes, and allowing for drift-wave type modes. Some further relation with engineering parameters can be extracted from equation (11). For simplicity, assume that $\lambda_{q} \sim R_{0}^{0.75} q^{0.75} B_{\phi}^{-0.25}$. Then, introducing $I_{\mathrm{p}} \sim a B_{\theta}$ we obtain $\lambda_{q} \sim B_{\phi}^{0.5}\left(I_{\mathrm{p}} / a^{2}\right)^{-0.75}$.

The SOL transport model used in this work involves resistive turbulent modes and sonic flows towards the limiter, both of which are strongly affected by the local temperature and density. In the end, it is fortuitous that the dependence on density and temperature almost vanishes from the final result. As an exercise, we have computed yet another formula for $\lambda_{q}$ by eliminating $n_{\mathrm{LCFS}}$ and $T_{e, \mathrm{LFCS}}$ dependence in equation (11). This allows us to apply the result of this work to the entire database. The resulting formula is

$$
\lambda_{q, \mathrm{fit}, 2}=8.7 \times 10^{-3} R_{0}^{0.73} q^{0.76} B_{\phi}^{-0.29}(\mathrm{~m}) .
$$

Eliminating the local temperature and density is not justified from a physical point of view and, furthermore, the indicial exponents of $\nu$ and $\rho_{\star}$ exceed their confidence intervals. In spite of this fact, equation (12) results in the same $R^{2}$ values as equation (11) when applied to the entire database. Thus, we conclude that $\lambda_{q}$ can be estimated using these formulas even if there is large uncertainty on $n_{\mathrm{LCFS}}$ and $T_{e, \mathrm{LCFS}}$, or even in their absence.

Finally, we stress that this exercise has demonstrated that a turbulent transport theory involving non-linearly saturated turbulence and sheath losses can describe the dependence 
of $\lambda_{q}$ with respect to the plasma parameters found in a large experimental database. Future avenues of research will concentrate on understanding plasma shaping effects (including the addition of an X-point), and evaluating the effects of turbulent transport on the formation of the near-SOL narrow heat-flux feature.

\section{Acknowledgments}

We would like to thank J Adamek, G Arnoux, J-G Bak, S Brezinsek, M Dimitrova, JP Gunn, J Havlicek, S-H Hong, F Janky, B LaBombard, S Marsen, G Maddaluno, L Nie, V Pericoli, Tsv Popov, R Panek, D Rudakov, J Seidl, DS Seo, M Shimada, C Silva, PC Stangeby, B Viola, P Vondracek, $\mathrm{H}$ Wang, GS Xu, and Y Xu for kindly providing the plasma profiles and the local data measurements necessary to carry out the analysis. We also thank S Jolliet, J Loizu, M Kočan, A Mosetto, F Riva, C Wersal and T-M Tran for useful discussions, and D Brunetti for carefully reading this manuscript. This research was supported in part by the Swiss National Science Foundation. We also acknowledge support from the Czech Science Foundation, project P205/12/2327. This work has been carried out within the framework of the EUROfusion Consortium and has received funding from the Euratom research and training programme 2014-2018 under grant agreement No 633053. The views and opinions expressed herein do not necessarily reflect those of the European Commission or those of the ITER Organization (Nuclear Facility INB no. 174).

\section{Appendix. Drift-reduced Braginskii equations}

Forcompleteness, weincludehere the drift-reducedBraginskii equations used as a basis for the transport analysis. The derivation of these model equations was first presented in [20]. Starting from the two Braginskii fluid equations [35], we impose the orderings $\mathrm{d} / \mathrm{d} t \ll \omega_{c i}, k_{\perp} \gg k_{\|}, \beta \ll 1$. The neglect of magnetic flutter is justified by the low $\beta$ at the LCFS in the discharges studied. We consider a cold ion model, as discussed in section 2. Finite ion temperature effects are quantified in [17]; the principal result being that curvature-driven modes (i.e. resistive ballooning modes) are slightly enhanced with respect to the cold ion model. Furthermore, since the equations are used in a quasi-linear analysis, we include here only those collisional terms that have a noticeable effect on the linear stability-namely the thermal force and the parallel Spitzer resistivity. The driftreduced equations, in normalized units, read as follows:

$$
\begin{aligned}
& \partial_{t} n=-\frac{\rho_{\star}^{-1}}{B}[\phi, n]-\nabla_{\|}\left(n v_{\| e}\right)+\frac{2}{B}\left[\hat{C}\left(p_{e}\right)-n \hat{C}(\phi)\right] \\
& \partial_{t} \omega=-\frac{\rho_{\star}^{-1}}{B}[\phi, \omega]-v_{\| i} \nabla_{\|} \omega+\frac{2 B}{n} \hat{C}\left(p_{e}\right)+\frac{B^{2}}{n} \nabla_{\|} j_{\|}
\end{aligned}
$$

$$
\begin{aligned}
\partial_{t} v_{\| e}= & -\frac{\rho_{\star}^{-1}}{B}\left[\phi, v_{\| e}\right]-v_{\| e} \nabla_{\|} v_{\| e} \\
& +\frac{m_{i}}{m_{e}}\left(\nu \frac{j_{\|}}{n}+\nabla_{\|} \phi-\frac{1}{n} \nabla_{\|} p_{e}-0.71 \nabla_{\|} T_{e}\right)
\end{aligned}
$$

$$
\partial_{t} v_{\| i}=-\frac{\rho_{\star}^{-1}}{B}\left[\phi, v_{\| i}\right]-v_{\| i} \nabla_{\|} v_{\| i}-\frac{1}{n} \nabla_{\|} p_{e}
$$

$$
\begin{aligned}
\partial_{t} T_{e}= & -\frac{\rho_{\star}^{-1}}{B}\left[\phi, T_{e}\right]-v_{\| e} \nabla_{\|} T_{e} \\
& +\frac{4}{3} \frac{T_{e}}{B}\left[\frac{7}{2} \hat{C}\left(T_{e}\right)+\frac{T_{e}}{n} \hat{C}(n)-\hat{C}(\phi)\right] \\
& +\frac{2}{3} T_{e}\left(\frac{0.71}{n} \nabla_{\|} j_{\|}-\nabla_{\|} v_{\| e}\right)
\end{aligned}
$$

where $\omega=\nabla_{\perp}^{2} \phi$ is the vorticity and equation (A.2) has been simplified using the Boussinesq approximation $\nabla \cdot\left(n d_{t} \nabla_{\perp} \phi\right) \approx n d_{t} \nabla_{\perp}^{2} \phi$. The parallel current is given by $j_{\|}=n\left(v_{\| i}-v_{\| e}\right)$. In addition, $[f, g]=\mathbf{B} \cdot(\nabla f \times \nabla g) / B$ is the Poisson bracket, while $\hat{C}(f)=(B / 2)\left[\nabla \times\left(\mathbf{B} / B^{2}\right)\right] \cdot \nabla f$ is the curvature operator. The (scalar) parallel derivative can be expressed as $\nabla_{\|} f=\left(\partial / \partial \varphi+q^{-1} \partial / \partial \theta\right) f$.

The reference units used to normalize the equations are $R_{0} / c_{s}$ (time), $\rho_{s}$ (perpendicular length), $R_{0}$ (parallel length), $B_{\phi}$ (magnetic field), $T_{e 0}$ (temperature), $n_{0}$ (density), and $e / T_{e 0}$ (potential). The major radius $R$ and the magnetic field $B_{\phi}$ are defined at the magnetic axis, while $n_{0}$ and $T_{e 0}$ are local quantities defined at the LCFS. The ion sound Larmor gyroradius $\rho_{s}$ is defined using $T_{e 0}$ and $B_{\phi}$. The dimensionless parameters $\rho_{\star}$ and $\nu$ are defined in equations (1) and (2).

We consider a circular plasma geometry with a toroidal limiter set at the high-field side equatorial mid plane, with the curvature terms described using the typical $\hat{s}-\alpha$ metric [36]. The quasi-linear transport solver considers a linearized version of these equations [31] to evaluate the required flux $\sim \gamma / k_{\theta}$.

\section{References}

[1] Pitts R A et al 2011 Physics basis and design of the ITER plasma-facing components $J$. Nucl. Mater. 415 S957-64

[2] Loarte A, the ITPA Scrape-off Layer and Divertor Physics Topical Group 2007 Chapter 4: Power and particle control Nucl. Fusion 47 S203

[3] Gunn J P et al 2013 Scrape-off layer power flux measurements in the Tore Supra tokamak J. Nucl. Mater. 438 S184-8

[4] Kočan M and Gunn J P 2010 Comparison of scrape-off layer profiles in outboard-versus inboard-limited plasmas in Tore Supra Plasma Phys. Control. Fusion 52045010

[5] Rudakov D L, Boedo J A, Pitts R A, Jackson G L, Lasnier C J, Leonard A W, Moyer R A, Stangeby P C, Tynan G R and Watkins J G 2011 SOL width in limited versus diverted discharges in DIII-D J. Nucl. Mater. 415 S387-90 
[6] Silva C et al and JET-EFDA Contributors 2014 Characterization of scrape-off layer transport in jet limiter plasmas Nucl. Fusion 54083022

[7] Arnoux G et al and JET-EFDA Contributors 2013 Scrape-off layer properties of ITER-like limiter start-up plasmas in JET Nucl. Fusion $\mathbf{5 3} 073016$

[8] Horacek J et al 2014 Narrow heat flux channels in the COMPASS limiter scrape-off layer J. Nucl. Mater. $463385-8$

[9] Nespoli F, Labit B, Furno I, Canal G P and Fasoli A 2014 Heat loads in inboard limited l-mode plasmas in TCV J. Nucl. Mater. 463 393-6

[10] Dejarnac R et al 2015 Understanding narrow SOL power flux component in COMPASS limiter plasmas by use of Langmuir probes $J$. Nucl. Mater. 463 381-4

[11] Stangeby P C, Tsui C K, Lasnier C J, Boedo J A, Elder J D, Kocan M, Leonard A W, McLean A G, Pitts R A and Rudakov D L 2015 Power deposition on the DIII-D inner wall limiter J. Nucl. Mater. 463 389-92

[12] Kocan M et al 2015 Impact of a narrow limiter SOL heat flux channel on the ITER first wall panel shaping Nucl. Fusion $\mathbf{5 5} 033019$

[13] Horacek J et al 2016 Plasma Phys. Control. Fusion 58074005

[14] Ricci P and Rogers B N 2013 Plasma turbulence in the scrape-off layer of tokamak devices Phys. Plasmas 20010702

[15] Halpern F D et al and JET-EFDA Contributors 2013 Theorybased scaling of the SOL width in circular limited tokamak plasmas Nucl. Fusion $\mathbf{5 3} 122001$

[16] Mosetto A, Halpern F D, Jolliet S, Loizu J and Ricci P 2013 Turbulent regimes in the tokamak scrape-off layer Phys. Plasmas 20092308

[17] Mosetto A, Halpern F D, Jolliet S, Loizu J and Ricci P 2015 Finite ion temperature effects on scrape-off layer turbulence Phys. Plasmas 22012308

[18] Halpern F D, Jolliet S, Loizu J, Mosetto A and Ricci P 2013 Ideal ballooning modes in the tokamak scrape-off layer Phys. Plasmas 20052306

[19] Halpern F D, Ricci P, Jolliet S, Loizu J and Mosetto A 2014 Theory of the scrape-off layer width in inner-wall limited tokamak plasmas Nucl. Fusion 54043003

[20] Zeiler A, Drake J F and Rogers B 1997 Nonlinear reduced Braginskii equations with ion thermal dynamics in toroidal plasma Phys. Plasmas 4 2134-8

[21] Ricci P, Halpern F D, Jolliet S, Loizu J, Mosetto A, Fasoli A, Furno I and Theiler C 2012 Simulation of plasma turbulence in scrape-off layer conditions: the GBS code, simulation results and code validation Plasma Phys. Control. Fusion 54124047

[22] Kočan M et al 2011 Measurements of ion energies in the tokamak plasma boundary J. Nucl. Mater. 415 S1133-8

[23] Manz P, Birkenmeier G, Carralero D, Fuchert G, Müller H W, Müller S H, Scott B D, Stroth U, Ribeiro T T and Wolfrum E 2015 The influence of finite ion temperature on plasma blob dynamics Plasma Phys. Control. Fusion 57014012

[24] Stangeby P C 2010 A three-dimensional analytic model for discrete limiters in ITER Nucl. Fusion 50035013

[25] LaBombard B 2015 private communication

[26] Goldston R J 2015 Theoretical aspects and practical implications of the heuristic drift SOL model J. Nucl. Mater. 463 397-400

[27] Eich T, Sieglin B, Scarabosio A, Fundamenski W, Goldston R J and Herrmann A 2011 Inter-ELM power decay length for JET and ASDEX upgrade: measurement and comparison with heuristic drift-based model Phys. Rev. Lett. 107215001

[28] Halpern F D 2015 Turbulence simulations of the narrow heatflux feature in inner-wall limited tokamaks Bull. Am. Phys. Soc. 60 VI2.00004

[29] Myra J R, D'Ippolito D A and Russell D A 2015 Turbulent transport regimes and the scrape-off layer heat flux width Phys. Plasmas 22042516

[30] Militello F, Naulin V and Nielsen A H 2013 Numerical scalings of the decay lengths in the scrape-off layer Plasma Phys. Control. Fusion 55074010

[31] Mosetto A, Halpern F D, Jolliet S and Ricci P 2012 Lowfrequency linear-mode regimes in the tokamak scrape-off layer Phys. Plasmas 19112103

[32] Meer P, Mintz D, Rosenfeld A and Kim D Y 1991 Robust regression methods for computer vision: a review Int. J. Comput. Vis. 6 59-70

[33] Kendl A and Scott B D 2006 Flux-surface shaping effects on tokamak edge turbulence and flows Phys. Plasmas 13012504

[34] Connor J W, Counsell G F, Erents S K, Fielding S J, LaBombard B and Morel K 1999 Comparison of theoretical models for scrape-off layer widths with data from COMPASS-D, JET and Alcator C-Mod Nucl. Fusion 39169

[35] Braginskii S I 1965 Transport Processes in a Plasma (Reviews of Plasma Physics vol 1) (New York: Consultants Bureau)

[36] Connor J W, Hastie R J and Taylor J B 1978 Shear, periodicity and plasma ballooning modes Phys. Rev. Lett. 40 396-9 